\title{
Synergistic Effect of Pd(II) and Acid Catalysts on Tandem Annulation Reaction for the Regioselective Synthesis of Ring-Fused Quinolines ${ }^{\dagger}$
}

\author{
Su Kyung Ock ${ }^{\ddagger}$ and So Won Youn* \\ Department of Chemistry, Hanyang University, Seoul 133-791, Korea. *E-mail: sowony73@hanyang.ac.kr \\ ${ }^{\ddagger}$ Department of Chemistry, Pukyong National University, Busan 608-737, Korea \\ Received October 15, 2009, Accepted October 25, 2009
}

Key Words: Tandem annulation reaction, Quinolines, $\mathrm{Pd}(\mathrm{OAc})_{2}, p-\mathrm{TsOH}$

Transition-metal-catalyzed tandem annulations are powerful methods for the synthesis of a wide range of carbocycles and heterocycles in a convergent way. ${ }^{1}$ Molecules having two or more different unsaturated bonds are particularly interesting substrates for the construction of a variety of cyclic compounds not only involving multiple $\mathrm{C}-\mathrm{C}$ or $\mathrm{C}-\mathrm{X}$ bond formations with a single catalyst in one operation but also allowing for multiple mechanistic possibilities, which in turn may lead to different products.

In 2004, Yamamoto and co-workers reported a Cu-catalyzed tandem reaction between 2-alkynyl- $N$-arylideneanilines and alcohols to afford $N$-(alkoxybenzyl)indoles. ${ }^{2}$ In contrast, Zhu et al. demonstrated that the regioselective synthesis of 4-alkoxy2-arylquinolines has been achieved by acid-catalyzed reaction of 2-(2-(trimethylsilyl)ethynyl)aniline, arylaldehydes, and alcohols. ${ }^{3}$ In Yamamoto's work, $\mathrm{CuCl}$ acts as a transition-metal catalyst which activates the alkyne moiety to first promote the intramolecular addition of the nitrogen atom of the imine to the activated alkynes, allowing the synthesis of indoles (Scheme 1, path a). On the other hand, acid catalyst activates the imine moiety which first undergoes the intramolecular attack of the alkyne, leading to quinolines (Scheme 1, path b).

The corresponding intramolecular version of these reactions could provide the ring-fused $N$-heterocycles. It has been shown that carbophilic $\mathrm{Au}(\mathrm{III})$ salts can catalyze the tandem reaction of $N$-(o-alkynylphenyl)imine (A) which has a tethered nucleophile, $\mathrm{OH}$ group, in the molecule, to give ring-fused indoles (Scheme 2, path a). Interestingly, iodonium-mediated reaction of the same substrates provided either ring-fused indoles or quinolines depending on the reaction conditions (Scheme 2, path a or b). ${ }^{5}$

Since the reactivity of the same substrates could be modulated to afford different outcomes through an alternative reaction pathway by tuning metal catalysts and/or reaction conditions, we envisioned that $N$-(o-alkynylphenyl)imine (A) might be cyclized to give ring-fused quinoline derivatives through the following mechanism; either via the intramolecular attack of the alkyne moiety to iminium ion, followed by trapping the vinyl cationic intermediate with a tethered nucleophile in a stepwise mechanism or by a concerted mechanism in which intramolecular addition of a tethered nucleophile to the activated alkyne upon coordination to a metal catalyst is followed by subsequent

${ }^{\dagger}$ This paper is dedicated to Professor Sunggak Kim on the occasion of his honorable retirement. nucleophilic attack to the iminium ion. Quinolines are found to possess a wide range of biological activities. ${ }^{6}$ In parallel with our efforts to develop a catalytic system for heterocyclic synthesis, ${ }^{7}$ we were interested in developing a one-pot synthesis of quinolines. Herein we report a new tandem reaction of $o$-alkynylanilines bearing $\mathrm{OH}$ moiety placed at an appropriate position with aldehydes catalyzed by the combination of $\mathrm{Pd}(\mathrm{OAc})_{2}$ and $p-\mathrm{TsOH}$ for the regioselective synthesis of ring-fused quinolines.

We focused our initial efforts on establishing optimal conditions for the tandem cyclization reactions between 1a and benzaldehyde, which were selected as the first substrates for screening of several metal salts and complexes, additives, and solvents. Based on the mechanisms established for the related tandem reactions, ${ }^{3,5}$ the success of a tandem cyclization triggered by the intramolecular attack of the alkyne to the iminium ion could be achieved by choosing the more Lewis acidic metal salts which have the more bias to activate the imine moiety than the alkyne functionality, resulting in both the enhancement of the electrophilicity of the imine and the decreased nucleophilicity of the nitrogen atom of the imine, while suppressing or preventing the intramolecular attack of imine to alkyne.

In accord with this hypothesis, Lewis acids such as Fe(III) and $\mathrm{Sc}(\mathrm{III})$ promoted the tandem reaction to give the desired

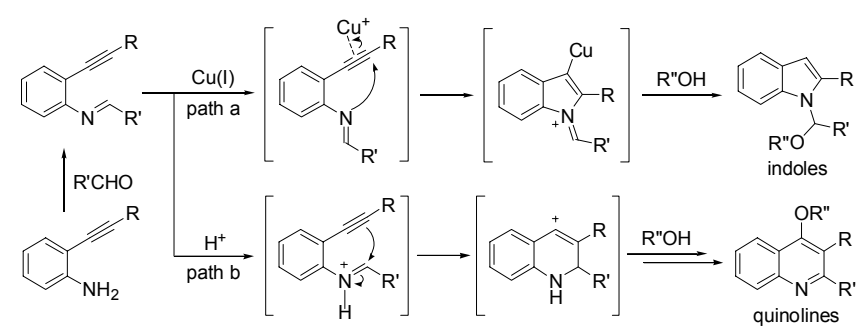

Scheme 1

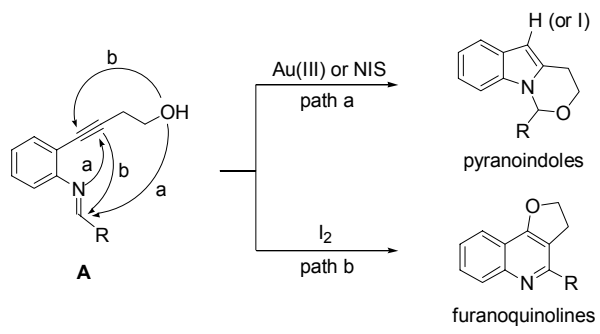

Scheme 2 
Table 1. Selected data from a systematic screening for the optimizations of reaction conditions for the synthesis of furanoquinoline $\mathbf{2} \mathbf{a}^{a}$

\begin{tabular}{|c|c|c|c|}
\hline & 1a & & \\
\hline entry & catalyst & additive & yield $(\%)^{b}$ \\
\hline 1 & $\mathrm{FeCl}_{3} / \mathrm{AgOTf}$ & - & 35 \\
\hline $2^{c}$ & $\mathrm{Sc}(\mathrm{OTf})_{3}$ & - & 25 \\
\hline $3^{c}$ & $\mathrm{RhCl}\left(\mathrm{PPh}_{3}\right)_{3} / \mathrm{AgOTf}$ & - & 35 \\
\hline 4 & $\mathrm{PtCl}_{2} / \mathrm{AgOTf}$ & - & 30 \\
\hline $5^{c}$ & $\mathrm{Ph}_{3} \mathrm{PAuCl} / \mathrm{AgOTf}$ & - & 35 \\
\hline $6^{c}$ & $\mathrm{AuCl}_{3} / \mathrm{AgOTf}$ & - & 15 \\
\hline $7^{c}$ & AgOTf & - & - \\
\hline $8^{d}$ & $\mathrm{Pd}(\mathrm{OAc})_{2}$ & - & 10 \\
\hline 9 & $\mathrm{FeCl}_{3} / \mathrm{AgOTf}$ & $\mathrm{CF}_{3} \mathrm{CO}_{2} \mathrm{H}$ & 50 \\
\hline $10^{c}$ & $\mathrm{RhCl}\left(\mathrm{PPh}_{3}\right)_{3} / \mathrm{AgOTf}$ & $\mathrm{CF}_{3} \mathrm{CO}_{2} \mathrm{H}$ & $55(50)^{d}$ \\
\hline 11 & $\mathrm{PtCl}_{2} / \mathrm{AgOTf}$ & $\mathrm{CF}_{3} \mathrm{CO}_{2} \mathrm{H}$ & 30 \\
\hline $12^{c}$ & $\mathrm{Ph}_{3} \mathrm{PAuCl} / \mathrm{AgOTf}$ & $\mathrm{CF}_{3} \mathrm{CO}_{2} \mathrm{H}$ & 30 \\
\hline $13^{c}$ & $\mathrm{AuCl}_{3} / \mathrm{AgOTf}$ & $\mathrm{CF}_{3} \mathrm{CO}_{2} \mathrm{H}$ & 15 \\
\hline $14^{d}$ & $\mathrm{Pd}(\mathrm{OAc})_{2}$ & $\mathrm{CF}_{3} \mathrm{CO}_{2} \mathrm{H}$ & 85 \\
\hline $15^{d, e}$ & $\mathrm{Pd}(\mathrm{OAc})_{2}$ & $\mathrm{AcOH}$ & - \\
\hline $16^{d, e}$ & $\mathrm{Pd}(\mathrm{OAc})_{2}$ & $\mathrm{MsOH}$ & 75 \\
\hline $17^{d}$ & $\mathrm{Pd}(\mathrm{OAc})_{2}$ & $p$-TsOH & 90 \\
\hline 18 & - & $\mathrm{CF}_{3} \mathrm{CO}_{2} \mathrm{H}$ & 35 \\
\hline 19 & - & $p$-TsOH & 30 \\
\hline
\end{tabular}

${ }^{a}$ Reaction conditions: 1a (1 equiv), PhCHO (1 equiv), catalyst (5 mol \%), and additive (20 mol \%) in $\mathrm{ClCH}_{2} \mathrm{CH}_{2} \mathrm{Cl}(0.05 \mathrm{M})$ at $80^{\circ} \mathrm{C}$ for $24 \mathrm{~h}$, unless otherwise noted. ${ }^{b}$ Determined by ${ }^{1} \mathrm{H}$ NMR using trichloroethylene as an internal standard. ${ }^{c}$ Performed with $4 \mathrm{~mol} \%$ of catalyst. ${ }^{d}$ Performed with 1.5 equiv of $\mathrm{PhCHO} .{ }^{e}$ Performed for 12 hours.

furanoquinoline $2 \mathbf{a}$, albeit only in $25 \sim 35 \%$ yields (Table 1 , entries 1-2). Interestingly, however, $\mathrm{Rh}(\mathrm{I}), \mathrm{Pt}(\mathrm{II})$, and $\mathrm{Au}(\mathrm{I})$ also afforded the similar results (Table 1, entries 3-5), whereas $\mathrm{Au}(\mathrm{III})$ gave the poorer yield as expected due to its high carbophilicity (Table 1 , entry 6). ${ }^{8}$ In contrast, other Lewis acids such as $\mathrm{Cu}(\mathrm{II}), \mathrm{Ag}(\mathrm{I}), \mathrm{Pd}(\mathrm{II}), \mathrm{Zn}(\mathrm{II})$, and In(III) were not effective. To boost the reactivity by increasing the electrophilicity of imine more efficiently, acid additives including organic and inorganic acids such as $\mathrm{AcOH}, \mathrm{CF}_{3} \mathrm{CO}_{2} \mathrm{H}, p$-TsOH, $\mathrm{MsOH}, \mathrm{HCl}, \mathrm{H}_{2} \mathrm{SO}_{4}$, etc. as well as Lewis acids such as $\mathrm{AlCl}_{3}, \mathrm{ZnCl}_{2}, \mathrm{In}(\mathrm{OTf})_{3}, \mathrm{YbCl}_{3}$, etc. were added to the reaction mixture. With the exception of $\mathrm{Au}(\mathrm{I}), \mathrm{Au}(\mathrm{III})$, and Pt(II), most of the metal salts examined provided 2a in substantially improved yields upon the addition of catalytic amount (20 mol \%) of acids (Table 1, entries 9-14). Among various acids examined, $\mathrm{CF}_{3} \mathrm{CO}_{2} \mathrm{H}, \mathrm{MsOH}$, and $p$ - $\mathrm{TsOH}$ showed outstanding effects (Table 1 , entries $14 \& 16-17$ ), and control experiments employing only these acids as the sole catalyst gave lower yields ( $\sim 30 \%$ ) (Table 1, entries $18-19)$. These results indicate that there is a remarkable synergistic effect of metal and acid catalysts which can obviously increase the reactivity of the substrate for this tandem cyclization reaction, whereas either catalyst alone is not active enough under the same conditions.

Finally, we were delighted to identify an exciting lead, which unambiguously stood out in the array of experiments. The com-
Table 2. $\mathrm{Pd}(\mathrm{OAc})_{2} / p$-TsOH-catalyzed synthesis of a variety of quinolines ${ }^{a}$
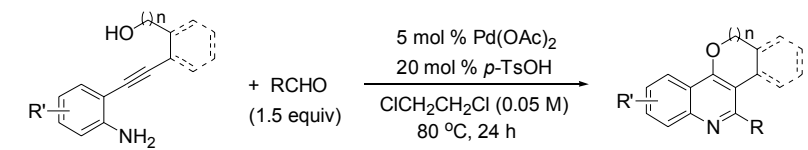

$1(n=0,1)$

2
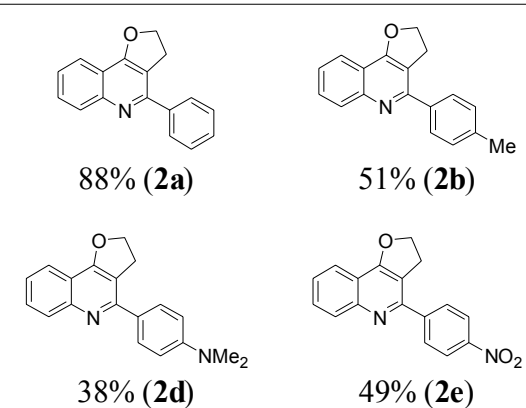

$51 \%(2 \mathbf{b})$

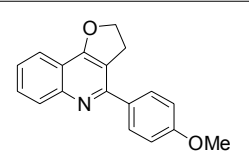

$55 \%(2 \mathrm{c})$
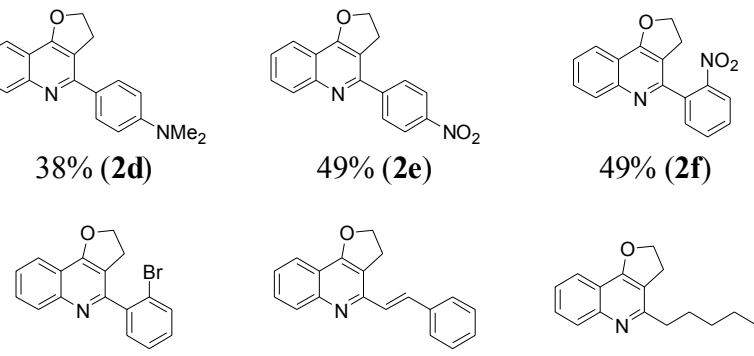

$49 \%$ (2f)

46\% (2g)

$25 \%$ (2h)

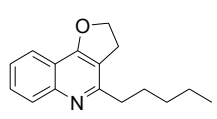

17\% (2i)
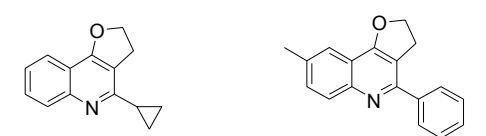

$58 \%(2 \mathbf{k})$

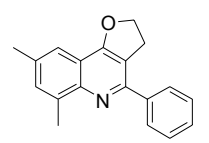

$62 \%(21)$
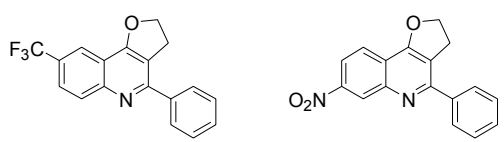

$58 \%$ (2n)

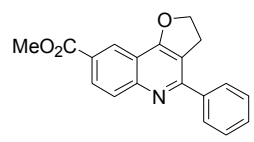

$65 \%(2 \mathrm{~m})$

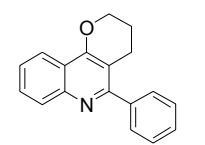

38\% (20)

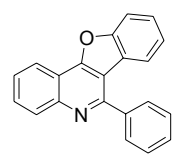

$52 \%(2 p)$

$11 \%, 30 \%{ }^{b}(\mathbf{2 q})$

${ }^{a}$ Isolated yields. ${ }^{b}$ Performed with 5 mol \% FeCl $3 /$ AgOTf for 29 hours.

bination of $5 \mathrm{~mol} \% \mathrm{Pd}(\mathrm{OAc})_{2}$ and $20 \mathrm{~mol} \%$ - $\mathrm{TsOH}$ was opti$\mathrm{mal}$ in this reaction system to produce $\mathbf{2} \mathbf{a}$ as a sole product in $90 \%$ yield (Table 1 , entry 17 ). Increasing the amount of either metal catalyst or acid catalyst and reaction temperature caused slightly lower yields or marginal effect. Various solvents were examined, and dichloroethane appeared preferable.

With the establishment of these optimized conditions, we set out to explore the scope of this tandem process (Table 2). No successful results have not been reported employing either highly electron-deficient arylaldehydes, such as 4-nitrobenzaldehyde, or alkylaldehydes in the previously reported related tandem cyclization reactions. ${ }^{3-5}$ In contrast, in our reaction system both electron-rich and electron-deficient arylaldehydes as well as sterically hindered arylaldehydes underwent tandem cyclization reaction to form the corresponding quinolines (Table 2, 2a-2g). Moreover, cyclopropanecarboxaldehyde was also suitable substrate (Table 2, 2j), whereas cinnamaldehyde and 
hexanal were not successful to give the corresponding products in good yields (Table 2, 2h-2i).

We proceeded to examine the effects of substituent R' on the aniline ring. A variety of aniline derivatives with electron-donating or -withdrawing substituents at various positions were found to be good substrates in this reaction (Table 2, 2k-2o).

Next, we tried to change the substituent at the end of the alkyne moiety. The reaction of 2-hydroxyphenyl-substituted aniline derivative proceeded smoothly to give the corresponding benzofuranoquinoline 2p in 52\% yield. Unfortunately, however, this transformation could not be successfully extended to the substrate with a longer carbon tether for the synthesis of the corresponding pyranoquinoline $\mathbf{2 q}$, although replacing $\mathrm{Pd}(\mathrm{II})$ by $\mathrm{Fe}(\mathrm{III})$ provided slightly improved yield.

In most reactions, analysis of the crude reaction mixture by ${ }^{1} \mathrm{H}$ NMR showed the remainder of the material to be either 2-(4,5-dihydrofuran-2-yl)anilines or $N$-benzylidene-2-(4,5-dihydrofuran-2-yl)anilines which could be both formed via only intramolecular addition of hydroxyl group to alkyne moiety. It should be noted that the corresponding indole products were not observed. Increasing either catalyst loadings or reaction temperature could not improve yields of products but only resulted in shorter reaction time.

In summary, we have developed tandem cyclization reaction of $o$-alkynylanilines and aldehydes to afford ring-fused quinolines. The combination of $\mathrm{Pd}(\mathrm{II})$ and protic acid catalysts exhibited synergistic effect to improve the efficiency of this tandem cyclization. Rational design of catalytic system and exquisite tune of catalysts and reaction conditions could modulate the reaction propensity and reaction pathway for the regioselective synthesis of quinoline derivatives. In no case were the corresponding indole products formed as a byproduct of this reaction. Although more detailed investigations are needed to clarify the mechanism for the present reaction, it is likely that this tandem reaction proceeds via a reaction pathway involving alkyne activation by coordination to $\mathrm{Pd}(\mathrm{II})$ as well as imine activation by acid alone or by acid and $\mathrm{Pd}(\mathrm{II})$ together. Noteworthy is the fact that this process can tolerate various functional groups such as methoxy, amino, bromo, $\mathrm{CF}_{3}$, alkene, cyclopropyl, nitro, and ester groups. In comparison with other related tandem reactions, ${ }^{4-5}$ imine preparation prior to tandem annulation is not required, and thus anilines and aldehydes can be used directly as substrates, obviating the need for pregeneration of imine in an additional step. Therefore, this regioselective one-pot reaction offers considerable advantages with regard to simplicity and high efficiency.

\section{Experimental Section}

General procedure for $\mathrm{Pd}(\mathrm{OAc})_{2} / p$-TsOH-catalyzed tandem cyclization reaction of $o$-alkynylanilines and aldehydes for the synthesis of ring-fused quinolines. To a solution of the substrate 1 in 1,2-dichloroethane $(0.05 \mathrm{M})$ were added $\mathrm{Pd}(\mathrm{OAc})_{2}(5 \mathrm{~mol} \%), p$-TsOH (20 mol \%), and aldehyde $(1.5$ equiv). The resulting mixture was stirred at the $80{ }^{\circ} \mathrm{C}$ for 24 hours. After the reaction was completed, the reaction mixture was quenched with distilled water, extracted with $\mathrm{CH}_{2} \mathrm{Cl}_{2}$ (three times), washed with brine, dried over $\mathrm{MgSO}_{4}$, and concentrated in vacuo. The residue was purified by column chromatography on silica gel (EtOAc : $n$-Hexane $=1: 10 \sim 1: 20$ ) to give the corresponding product 2 .

Spectral data for $\mathbf{2 a - 2 c}$ and $\mathbf{2 q}$ were consistent with data reported in the literature. ${ }^{5}$

4-(2,3-Dihydrofuro [3,2-c]quinolin-4-yl)- $\mathbf{N}, \mathbf{N}$-dimethylbenzenamine (2d): ${ }^{1} \mathrm{H} \mathrm{NMR}\left(400 \mathrm{MHz}\right.$, in $\left.\mathrm{CDCl}_{3}\right) \delta 3.03$ (s, $6 \mathrm{H}), 3.61(\mathrm{t}, J=8.9 \mathrm{~Hz}, 2 \mathrm{H}), 4.86(\mathrm{t}, J=8.9 \mathrm{~Hz}, 2 \mathrm{H}), 6.82$ (d, $J=8.9 \mathrm{~Hz}, 2 \mathrm{H}), 7.40(\mathrm{t}, J=7.5 \mathrm{~Hz}, 1 \mathrm{H}), 7.63(\mathrm{t}, J=7.7 \mathrm{~Hz}, 1 \mathrm{H})$, 7.87-7.92 (m, 3H), $8.08(\mathrm{~d}, J=8.9 \mathrm{~Hz}, 1 \mathrm{H}) ;{ }^{13} \mathrm{C} \mathrm{NMR}(100 \mathrm{MHz}$, in $\left.\mathrm{CDCl}_{3}\right) \delta 30.6,40.3,72.9,112.0,114.3,115.7,121.2,124.6$, $127.7,128.8,129.4,149.1,149.2,150.9,155.5,164.1 ; \mathrm{IR}(\mathrm{NaCl})$ 2919, 1607, 1590, 1501, 1363, 1196, 1085, 764, 694, $550 \mathrm{~cm}^{-1}$; EIMS $m / z 290\left(74, \mathrm{M}^{+}\right), 289\left(100, \mathrm{M}^{+}-\mathrm{H}\right), 273$ (28), 246 (13), 144 (15), 130 (11), 123 (10), 95 (4), 77 (3).

2,3-Dihydro-4-(4-nitrophenyl)furo[3,2-c]quinoline (2e): ${ }^{1} \mathrm{H}$ NMR (400 MHz, in $\left.\mathrm{CDCl}_{3}\right) \delta 3.61(\mathrm{t}, J=8.9 \mathrm{~Hz}, 2 \mathrm{H}), 4.94(\mathrm{t}$, $J=8.9 \mathrm{~Hz}, 2 \mathrm{H}), 7.52(\mathrm{t}, J=7.6 \mathrm{~Hz}, 1 \mathrm{H}), 7.71(\mathrm{t}, J=7.7 \mathrm{~Hz}$, $1 \mathrm{H}), 7.98(\mathrm{~d}, J=8.2 \mathrm{~Hz}, 1 \mathrm{H}), 8.11(\mathrm{~d}, J=8.9 \mathrm{~Hz}, 3 \mathrm{H}), 8.37(\mathrm{~d}$, $J=8.9 \mathrm{~Hz}, 2 \mathrm{H}) ;{ }^{13} \mathrm{C} \mathrm{NMR}\left(100 \mathrm{MHz}\right.$, in $\left.\mathrm{CDCl}_{3}\right) \delta 30.0,73.2$, $115.2,116.2,121.5,123.8,126.2,129.2,129.4,130.2,146.0$, 147.8, 149.2, 152.7, 164.9; IR (NaCl) 2923, 1556, 1519, 1344, 1089, 854, 766, $704 \mathrm{~cm}^{-1}$; EIMS m/z $292\left(89, \mathrm{M}^{+}\right), 291$ (84, $\left.\mathrm{M}^{+}-\mathrm{H}\right), 246(28), 245$ (100), 217 (25), 189 (8), $122(5), 108(25)$, 94 (12), 82 (7).

2,3-Dihydro-4-(2-nitrophenyl)furo[3,2-c]quinoline (2f): ${ }^{1} \mathrm{H}$ NMR (400 MHz, in $\left.\mathrm{CDCl}_{3}\right) \delta 3.23(\mathrm{t}, J=8.9 \mathrm{~Hz}, 2 \mathrm{H}), 4.89(\mathrm{t}$, $J=8.9 \mathrm{~Hz}, 2 \mathrm{H}), 7.51(\mathrm{t}, J=7.5 \mathrm{~Hz}, 1 \mathrm{H}), 7.58-7.62(\mathrm{~m}, 2 \mathrm{H})$, 7.65-7.75 (m, 2H), $7.98(\mathrm{~d}, J=8.2 \mathrm{~Hz}, 1 \mathrm{H}), 8.04(\mathrm{~d}, J=8.6 \mathrm{~Hz}$, $1 \mathrm{H}), 8.11(\mathrm{~d}, J=8.0 \mathrm{~Hz}, 1 \mathrm{H}) ;{ }^{13} \mathrm{C} \mathrm{NMR}\left(100 \mathrm{MHz}\right.$, in $\left.\mathrm{CDCl}_{3}\right)$ $\delta 28.4,73.2,116.0,116.2,121.5,124.6,125.9,129.2,129.4$, $129.9,131.2,133.2,135.2,148.4,148.9,153.4,164.0 ; \mathrm{IR}(\mathrm{NaCl})$ 2922, 2364, 1555, 1528, 1407, 1350, 1083, 915, 854, 766, 718, $694 \mathrm{~cm}^{-1}$; EIMS m/z $293\left(18, \mathrm{M}^{+}\right), 292\left(71, \mathrm{M}^{+}-\mathrm{H}\right), 262$ (100), 245 (32), 234 (24), 217 (98), 108 (29), 76 (20).

4-(2-Bromophenyl)-2,3-dihydrofuro[3,2-c]quinoline (2g): ${ }^{1} \mathrm{H} \mathrm{NMR}\left(400 \mathrm{MHz}\right.$, in $\left.\mathrm{CDCl}_{3}\right) \delta 3.29(\mathrm{t}, J=8.9 \mathrm{~Hz}, 2 \mathrm{H}), 4.89$ $(\mathrm{t}, J=8.9 \mathrm{~Hz}, 2 \mathrm{H}), 7.28-7.32(\mathrm{~m}, 1 \mathrm{H}), 7.42-7.46(\mathrm{~m}, 2 \mathrm{H}), 7.48-$ $7.52(\mathrm{~m}, 1 \mathrm{H}), 7.66-7.70(\mathrm{~m}, 2 \mathrm{H}), 7.99(\mathrm{~d}, J=8.2 \mathrm{~Hz}, 1 \mathrm{H}), 8.10$ $(\mathrm{d}, J=8.6 \mathrm{~Hz}, 1 \mathrm{H}) ;{ }^{13} \mathrm{C} \mathrm{NMR}\left(100 \mathrm{MHz}\right.$, in $\left.\mathrm{CDCl}_{3}\right) \delta 28.7,73.3$, 116.2, 116.8, 121.4, 121.7, 125.7, 127.6, 129.3, 129.7, 129.9, 130.4, 132.8, 141.0, 148.9, 156.3, 163.6; IR (NaCl) 2923, 1633, 1596, 1554, 1508, 1406, 1261, 1088, 1054, 1024, 914, 800, 762, 694, $644 \mathrm{~cm}^{-1}$; EIMS $\mathrm{m} / z 326\left(60, \mathrm{M}^{+}\right), 325$ (74, $\left.\mathrm{M}^{+}-\mathrm{H}\right), 246$ (100), 217 (62), 123 (20), 115 (18), 109 (52), 94 (22), 76 (12).

2,3-Dihydro-4-styrylfuro[3,2-c]quinoline (2h): ${ }^{1} \mathrm{H}$ NMR (400 $\mathrm{MHz}$, in $\left.\mathrm{CDCl}_{3}\right) \delta 3.59(\mathrm{t}, J=8.9 \mathrm{~Hz}, 2 \mathrm{H}), 4.95(\mathrm{t}, J=9.0 \mathrm{~Hz}$, $2 \mathrm{H}), 7.30-7.35(\mathrm{~m}, 2 \mathrm{H}), 7.38-7.45(\mathrm{~m}, 3 \mathrm{H}), 7.64-7.68(\mathrm{~m}, 3 \mathrm{H})$, $7.86(\mathrm{~d}, J=16.0 \mathrm{~Hz}, 1 \mathrm{H}), 7.90(\mathrm{dd}, J=0.7,8.2 \mathrm{~Hz}, 1 \mathrm{H}), 8.12$ $(\mathrm{d}, J=4.8 \mathrm{~Hz}, 1 \mathrm{H}) ;{ }^{13} \mathrm{C} \mathrm{NMR}\left(100 \mathrm{MHz}\right.$, in $\left.\mathrm{CDCl}_{3}\right) \delta 28.8,73.1$, 115.5, 116.1, 121.3, 125.1, 126.2, 127.3, 128.6, 128.7, 129.8, 135.2, 136.7, 149.1, 152.3, 164.0; IR (NaCl) 2923, 2369, 2343, $1594,1505,1259,1069,969,917,756,698 \mathrm{~cm}^{-1}$; EIMS m/z $273\left(86, \mathrm{M}^{+}\right), 272\left(100, \mathrm{M}^{+}-\mathrm{H}\right), 245$ (31), 217 (12), 196 (63), 167 (10), 120 (10), 115 (25), 77 (10).

2,3-Dihydro-4-pentylfuro[3,2-c]quinoline (2i): ${ }^{1} \mathrm{H} \mathrm{NMR}$ (400 $\mathrm{MHz}$, in $\left.\mathrm{CDCl}_{3}\right) \delta 0.91(\mathrm{t}, J=8.0 \mathrm{~Hz}, 3 \mathrm{H}), 1.33-1.45(\mathrm{~m}, 4 \mathrm{H})$, $1.74-1.81(\mathrm{~m}, 2 \mathrm{H}), 2.88(\mathrm{t}, J=7.9 \mathrm{~Hz}, 2 \mathrm{H}), 3.38(\mathrm{t}, J=9.1 \mathrm{~Hz}$, 
$2 \mathrm{H}), 4.88(\mathrm{t}, J=8.9 \mathrm{~Hz}, 2 \mathrm{H}), 7.41(\mathrm{t}, J=7.5 \mathrm{~Hz}, 1 \mathrm{H}), 7.62(\mathrm{t}$, $J=7.8 \mathrm{~Hz}, 1 \mathrm{H}), 7.89(\mathrm{dd}, J=0.7,8.2 \mathrm{~Hz}, 1 \mathrm{H}), 8.03(\mathrm{~d}, J=8.0$ $\mathrm{Hz}, 1 \mathrm{H}) ;{ }^{13} \mathrm{CNMR}\left(100 \mathrm{MHz}\right.$, in $\left.\mathrm{CDCl}_{3}\right) \delta 14.0,22.5,28.1,28.6$, $31.9,36.7,73.2,115.6,115.8,121.3,125.0,127.8,129.7,159.6$ $\mathrm{cm}^{-1}$; EIMS m/z $242\left(2, \mathrm{M}^{+}\right), 241\left(4, \mathrm{M}^{+}-\mathrm{H}\right), 226(1), 212(12)$, 198 (25), 185 (100), 167 (4), 130 (8), 102 (5).

4-Cyclopropyl-2,3-dihydrofuro[3,2-c] quinoline (2j): ${ }^{1} \mathrm{H}$ NMR (400 MHz, in $\left.\mathrm{CDCl}_{3}\right) \delta 1.01-1.05(\mathrm{~m}, 2 \mathrm{H}), 1.30(\mathrm{~s}, 2 \mathrm{H}), 2.01-$ $2.07(\mathrm{~m}, 1 \mathrm{H}), 3.46(\mathrm{t}, J=9.0 \mathrm{~Hz}, 2 \mathrm{H}), 4.88(\mathrm{t}, J=9.0 \mathrm{~Hz}, 2 \mathrm{H})$, $7.35(\mathrm{t}, J=7.5 \mathrm{~Hz}, 1 \mathrm{H}), 7.56(\mathrm{t}, J=7.7 \mathrm{~Hz}, 1 \mathrm{H}), 7.85(\mathrm{~d}, J=$ $8.2 \mathrm{~Hz}, 1 \mathrm{H}), 7.91(\mathrm{~d}, J=8.0 \mathrm{~Hz}, 1 \mathrm{H}) ;{ }^{13} \mathrm{C} \mathrm{NMR}(100 \mathrm{MHz}$, in $\left.\mathrm{CDCl}_{3}\right) \delta$ 9.3, 15.3, 28.1, 29.7, 73.0, 115.6, 115.8, 121.2, 124.2, 128.3, 129.1, 148.6, 159.8, 162.6; IR ( NaCl) 2919, 2352, 1595 , $1557,1505,1257,1079,1051,913,803,756,641 \mathrm{~cm}^{-1}$; EIMS $\mathrm{m} / \mathrm{z} 211\left(63, \mathrm{M}^{+}\right), 210\left(93, \mathrm{M}^{+}-\mathrm{H}\right), 196(100), 184(12), 167$ (33), 154 (12), 115 (11), 83 (12), 77 (15), 63 (11).

2,3-Dihydro-8-methyl-4-phenylfuro[3,2-c]quinoline (2k): ${ }^{1} \mathrm{H}$ NMR $\left(400 \mathrm{MHz}\right.$, in $\left.\mathrm{CDCl}_{3}\right) \delta 2.53(\mathrm{~s}, 3 \mathrm{H}), 3.57$ (t, $J=8.9$ $\mathrm{Hz}, 2 \mathrm{H}), 4.87(\mathrm{t}, J=8.9 \mathrm{~Hz}, 2 \mathrm{H}), 7.41-7.45(\mathrm{~m}, 1 \mathrm{H}), 7.48-7.52$ $(\mathrm{m}, 3 \mathrm{H}), 7.71(\mathrm{~s}, 1 \mathrm{H}), 7.89(\mathrm{dd}, J=3.2,5.3 \mathrm{~Hz}, 2 \mathrm{H}), 8.03(\mathrm{~d}$, $J=8.9 \mathrm{~Hz}, 1 \mathrm{H}) ;{ }^{13} \mathrm{C} \mathrm{NMR}\left(100 \mathrm{MHz}\right.$, in $\left.\mathrm{CDCl}_{3}\right) \delta 21.6,30.1$, 73.0, 115.0, 115.9, 120.0, 128.3, 128.5, 128.7, 128.9, 132.0, 135.3, 139.9, 147.7, 154.6, 163.8; IR (NaCl) 2923, 1636, 1599, 1555, 1494, 1402, 1332, 1197, 1086, 1040, 944, 824, 768, 700, $659 \mathrm{~cm}^{-1}$; EIMS m/z $261\left(64, \mathrm{M}^{+}\right), 260\left(100, \mathrm{M}^{+}-\mathrm{H}\right), 230$ (4), 217 (9), 189 (1), 130 (11), 115 (18), 89 (5), 77 (5).

2,3-Dihydro-6,8-dimethyl-4-phenylfuro[3,2-c]quinoline (2l): ${ }^{1} \mathrm{H} \mathrm{NMR}\left(400 \mathrm{MHz}\right.$, in $\left.\mathrm{CDCl}_{3}\right) \delta 2.48(\mathrm{~s}, 3 \mathrm{H}), 2.82(\mathrm{~s}, 3 \mathrm{H})$, $3.62(\mathrm{t}, J=8.9 \mathrm{~Hz}, 2 \mathrm{H}), 4.85(\mathrm{t}, J=8.9 \mathrm{~Hz}, 2 \mathrm{H}), 7.37(\mathrm{~s}, 1 \mathrm{H})$, $7.41-7.45(\mathrm{~m}, 1 \mathrm{H}), 7.50(\mathrm{t}, J=7.5 \mathrm{~Hz}, 2 \mathrm{H}), 7.57(\mathrm{~s}, 1 \mathrm{H}), 8.03$ (dd, $J=1.3,7.3 \mathrm{~Hz}, 2 \mathrm{H}) ;{ }^{13} \mathrm{C} \mathrm{NMR}\left(100 \mathrm{MHz}\right.$, in $\left.\mathrm{CDCl}_{3}\right) \delta 18.4$, 21.6, 30.5, 72.8, 114.6, 115.8, 117.8, 128.4, 128.5, 132.0, 134.8, 137.0, 140.4, 146.8, 152.5, 164.0; IR ( NaCl) 2919, 2374, 1599, 1492, 1399, 1336, 1209, 998, 926, 856, 770, $699 \mathrm{~cm}^{-1}$; EIMS $\mathrm{m} / z 275\left(100, \mathrm{M}^{+}\right), 274\left(97, \mathrm{M}^{+}-\mathrm{H}\right), 259$ (19), 230 (11), 137 (14), 130 (15), 115 (21), 77 (6).

8-(Trifluoromethyl)-2,3-dihydro-4-phenylfuro[3,2-c]quin oline (2m): ${ }^{1} \mathrm{H}$ NMR $\left(400 \mathrm{MHz}\right.$, in $\left.\mathrm{CDCl}_{3}\right) \delta 3.63(\mathrm{t}, J=8.9$ $\mathrm{Hz}, 2 \mathrm{H}), 4.94$ (t, $J=8.9 \mathrm{~Hz}, 2 \mathrm{H}), 7.46-7.55(\mathrm{~m}, 3 \mathrm{H}), 7.82$ (dd, $J=1.9,9.1 \mathrm{~Hz}, 1 \mathrm{H}), 7.92(\mathrm{dd}, J=1.3,7.2 \mathrm{~Hz}, 2 \mathrm{H}), 8.23(\mathrm{~d}, J=$ $8.9 \mathrm{~Hz}, 1 \mathrm{H}), 8.29(\mathrm{~s}, 1 \mathrm{H}) ;{ }^{13} \mathrm{CNMR}\left(100 \mathrm{MHz}\right.$, in $\left.\mathrm{CDCl}_{3}\right) \delta 30.1$, 73.5, 114.9, 116.4, 119.9, 119.9, 125.3, 128.3, 128.7, 129.4, 130.3, 139.2, 149.9, 157.4, 165.1; IR (NaCl) 2926, 2363, 1601, $1561,1343,1306,1267,1157,1105,840,697 \mathrm{~cm}^{-1}$; EIMS m/z $315\left(52, \mathrm{M}^{+}\right), 314\left(100, \mathrm{M}^{+}-\mathrm{H}\right), 285(7), 245(9), 217(12), 158$ (6), 133 (8), 108 (6), 77 (4).

2,3-Dihydro-7-nitro-4-phenylfuro[3,2-c]quinoline (2n): ${ }^{1} \mathrm{H}$ NMR (400 MHz, in $\left.\mathrm{CDCl}_{3}\right) \delta 3.66(\mathrm{t}, J=9.0 \mathrm{~Hz}, 2 \mathrm{H}), 4.96(\mathrm{t}$, $J=9.0 \mathrm{~Hz}, 2 \mathrm{H}), 7.48-7.57$ (m, 3H), 7.94 (d, $J=7.5 \mathrm{~Hz}, 2 \mathrm{H})$, $8.08(\mathrm{~d}, J=8.9 \mathrm{~Hz}, 1 \mathrm{H}), 8.22(\mathrm{dd}, J=2.2,9.1 \mathrm{~Hz}, 1 \mathrm{H}), 9.03(\mathrm{~s}$, $1 \mathrm{H}) ;{ }^{13} \mathrm{C}$ NMR $\left(100 \mathrm{MHz}\right.$, in $\left.\mathrm{CDCl}_{3}\right) \delta 30.3,73.6,118.3,118.6$, 119.0, 123.3, 125.5, 127.4, 128.3, 128.7, 129.1, 129.6, 138.9, 148.0, 148.5, 157.9, 164.3; IR ( NaCl) 2927, 2369, 1639, 1524, 1332, 1246, 1066, 738, $696 \mathrm{~cm}^{-1}$; EIMS $m / z 292\left(87, \mathrm{M}^{+}\right), 291$
(100, $\left.\mathrm{M}^{+}-\mathrm{H}\right), 245$ (99), 233 (22), 217 (33), 203 (11), 189 (19), 108 (27), 94 (34), 82 (15), 77 (20), 63 (18).

Methyl 2,3-dihydro-4-phenylfuro[3,2-c]quinoline-8-carboxylate (2o): ${ }^{1} \mathrm{H}$ NMR $\left(400 \mathrm{MHz}\right.$, in $\left.\mathrm{CDCl}_{3}\right) \delta 3.62(\mathrm{t}, J=8.9$ $\mathrm{Hz}, 2 \mathrm{H}), 3.98(\mathrm{~s}, 3 \mathrm{H}), 4.95(\mathrm{t}, J=9.1 \mathrm{~Hz}, 2 \mathrm{H}), 7.46-7.55(\mathrm{~m}$, $3 \mathrm{H}), 7.93$ (dd, $J=1.4,8.2 \mathrm{~Hz}, 2 \mathrm{H}), 8.18$ (s, 1H), 8.25 (dd, $J=$ $1.7,8.9 \mathrm{~Hz}, 1 \mathrm{H}), 8.74(\mathrm{~d}, J=2.4 \mathrm{~Hz}, 1 \mathrm{H}) ;{ }^{13} \mathrm{C} \mathrm{NMR}(100 \mathrm{MHz}$, in $\left.\mathrm{CDCl}_{3}\right) \delta 29.7,30.0,52.3,73.5,115.1,116.0,125.0,126.7$, 128.4, 128.6, 129.3, 129.4, 139.1, 139.2, 150.6, 157.4, 165.7, 166.6; IR ( NaCl) 2927, 2374, 1706, 1635, 1263, 1115, 1077, $773,694 \mathrm{~cm}^{-1}$; EIMS m/z $305\left(77, \mathrm{M}^{+}\right), 304\left(100, \mathrm{M}^{+}-\mathrm{H}\right), 245$ (25), 217 (15), 189 (5), 152 (4), 123 (9), 108 (25), 94 (8), 77 (3).

6-Phenylbenzofuro[3,2-c] quinoline (2p): ${ }^{1} \mathrm{H} \mathrm{NMR}(400 \mathrm{MHz}$, in $\left.\mathrm{CDCl}_{3}\right) \delta 7.29(\mathrm{t}, J=7.6 \mathrm{~Hz}, 1 \mathrm{H}), 7.51(\mathrm{dt}, J=1.1,7.8 \mathrm{~Hz}$, 1H), 7.57-7.68 (m, 3H), $7.74(\mathrm{~d}, J=7.5 \mathrm{~Hz}, 2 \mathrm{H}), 7.77$ (d, $J=8.2$ $\mathrm{Hz}, 1 \mathrm{H}), 7.82(\mathrm{dt}, J=1.4,7.7 \mathrm{~Hz}, 1 \mathrm{H}), 7.96(\mathrm{dd}, J=1.4,8.9 \mathrm{~Hz}$, $2 \mathrm{H}), 8.37(\mathrm{~d}, J=8.2 \mathrm{~Hz}, 1 \mathrm{H}), 8.46(\mathrm{dd}, J=0.7,8.2 \mathrm{~Hz}, 1 \mathrm{H})$; ${ }^{13} \mathrm{C}$ NMR $\left(100 \mathrm{MHz}\right.$, in $\left.\mathrm{CDCl}_{3}\right) \delta 111.9,114.5,116.2,120.7$, 122.2, 122.8, 123.6, 126.7, 127.0, 128.7, 129.0, 129.4, 129.6, 129.7, 139.5, 146.8, 155.8, 156.0, $158.3 \mathrm{~cm}^{-1}$; EIMS m/z 295 $\left(71, \mathrm{M}^{+}\right), 294\left(100, \mathrm{M}^{+}-\mathrm{H}\right), 265$ (16), 239 (6), 147 (16), 132 (22), 119 (14), 94 (3).

Acknowledgments. This work was supported by National Research Foundation of Korea Grant funded by the Korean Government (No. R01-2008-000-20332-0 \& 2009-0063856). We are grateful to BK21 Foundation for generous support.

\section{References}

1. For reviews, see: (a) Montgomery, J. Angew. Chem., Int. Ed. 2004, 43, 3890. (b) Negishi, E.; Copéret, C.; Ma, S.; Liou, S. Y.; Liu, F. Chem. Rev. 1996, 96, 365. (c) Tietze, L. F. Chem. Rev. 1996, 96, 115. (d) Grigg, R.; Sridharan, V. J. Organomet. Chem. 1999, 576, 65. (e) Miura, T.; Murakami, M. Chem. Commun. 2007, 217. (f) Youn, S. W. Eur. J. Org. Chem. 2009, 2597. (g) Lee, J. M.; Na, Y.; Han, H.; Chang, S. Chem. Soc. Rev. 2004, 33, 302.

2. Kamijo, S.; Sasaki, Y.; Yamamoto, Y. Tetrahedron Lett. 2004, 45, 35 .

3. Wang, Y.; Peng, C.; Liu, L.; Zhao, J.; Su, L.; Zhu, Q. Tetrahedron Lett. 2009, 50, 2261.

4. Fu, W.; Xu, C.; Zou, G.; Hong, D.; Deng, D.; Wang, Z.; Ji, B. Synlett 2009, 763 .

5. Halim, R.; Scammells, P. J.; Flynn, B. L. Org. Lett. 2008, 10, 1967.

6. For reviews, see: (a) Sikorski, J. A. J. Med. Chem. 2006, $49,1$. (b) Bradburry, B. J.; Pucci, M. J. Curr. Opin. Pharm. 2008, 8, 574. (c) Michael, J. P. Nat. Prod. Rep. 2008, 25, 166.

7. (a) Pastine, S. J.; Youn, S. W.; Sames, D. Org. Lett. 2003, 5, 1055. (b) Pastine, S. J.; Youn, S. W.; Sames, D. Tetrahedron 2003, 59 , 8859. (c) Youn, S. W.; Pastine, S. J.; Sames, D. Org. Lett. 2004, 6, 581. (d) Youn, S. W.; Eom, J. I. Org. Lett. 2005, 7, 3355. (e) Youn, S. W. J. Org. Chem. 2006, 71, 2521. (f) Youn, S. W. Org. Prep. Proced. Int. 2006, 38, 505. (g) Youn, S. W.; Eom, J. I. J. Org. Chem. 2006, 71, 6705. (h) Youn, S. W. Synlett 2007, 3050. (i) Youn, S. W.; Song, J.-H.; Jung, D.-I. J. Org. Chem. 2008, 73, 5658. (j) Youn, S. W.; Bihn, J. H. Tetrahedron Lett. 2009, 50, 4598.

8. In general, the use of AgOTf along with metal salts such as $\mathrm{FeCl}_{3}$, $\mathrm{RhCl}\left(\mathrm{PPh}_{3}\right)_{3}, \mathrm{PtCl}_{2}, \mathrm{Ph}_{3} \mathrm{PAuCl}$, and $\mathrm{AuCl}_{3}$ afforded the better yields of the product $\mathbf{2 a}$ than use of only these metal salts. 